\title{
Continuous intrathecal administration of liposomal amphotericin B for treatment of refractory Cryptococcus neoformans encephalitis: A case report
}

\author{
HAITING XIE*, PENG LUO*, ZHONGLI LI, RUI LI, HAITAO SUN and DUOBIN WU \\ Department of Neurology, Zhujiang Hospital of Southern Medical University, Guangzhou, Guangdong 510282, P.R. China
}

Received October 26, 2015; Accepted February 27, 2017

DOI: $10.3892 / \mathrm{etm} .2017 .4554$

\begin{abstract}
The aim of the present study was to investigate the treatment of refractory Cryptococcus neoformans encephalitis with continuous administration of liposomal amphotericin B (AmB). Liposomal AmB was administered to a 28-year-old male by intravenous injection, with daily increasing dosages up to $150 \mathrm{mg}$ per day and combined use of fluconazole ( $0.4 \mathrm{~g}$ per day) and oral flucytosine tablets (1.5 g per day). Following 5 months of treatment, $C$. neoformans could still be detected in the ink stain of cerebrospinal fluid, but the patient could not tolerate a further increase in the dosage of liposomal AmB. Instead, continuous intrathecal administration of AmB through tube drainage on the lumbar cistern was used. A total dosage of $28 \mathrm{mg}$ liposomal AmB was administered to the patient over the course of 1 month. The effect of AmB administered by intravenous injection was not as great as expected and the patient's tolerance was not good. However, the patient recovered following treatment by continuous intrathecal administration of AmB through tube drainage on the lumbar cistern for 1 month. This case suggests that continuous intrathecal administration of liposomal AmB should be considered for clinical treatment of refractory cryptococcal encephalitis.
\end{abstract}

\section{Introduction}

Cryptococcus neoformans encephalitis is a type of fungal infection in the central nervous system caused by C. neoformans (1). Approximately 6-10\% of AIDS patients in the USA and up to $30 \%$ in Africa are infected with $C$. neoformans, and 90\% develop meningoencephalitis (1). When AIDS patients with cryptococcal meningoencephalitis were treated

Correspondence to: Professor Duobin Wu, Department of Neurology, Zhujiang Hospital of Southern Medical University, 253 Industry Road, Guangzhou, Guangdong 510282, P.R. China E-mail:nfykdx@sina.com

*Contributed equally

Key words: liposomal amphotericin B, Cryptococcus neoformans, encephalitis with amphotericin B or fluconazole, the meningoencephalitis resolved in one-third, active meningoencephalitis persisted in one-third and approximately one-third died (2) In populations with normal immunological function, the infection rate of Cryptococcus is $\sim 1$ in 100,000 (3), and the incidence of life-threatening cryptococcal infections among patients with AIDS has been estimated at 6 to $10 \%$ in the United States, western Europe, and Australia, and 15 to $30 \%$ in sub-Saharan Africa (3). However the prevalence of this disease in healthy people is increasing year on year (4). Furthermore, outbreaks of cryptococcosis in which $>1$ patient acquires the infection at the same time and place, presumably from exposure to a common source of the inoculum, do not occur (5). During initial therapy, $10-25 \%$ of these patients die, and $30-60 \%$ succumb within 12 months (3).

AmB has been successfully used to treat every type of cryptococcosis, from pneumonia to meningitis; however, the only comparative trials have been in cases of meningitis (3). Because of its toxicity, AmB therapy has been reduced in dose and duration by successfully using it in combination with flucytosine (6). Formulations of AMB and lipids have been developed to aid its delivery, reduce toxicity, and enable higher doses to be tolerated (1). In an attempt to optimize therapy for cryptococcal meningitis, AMB has also been used locally at the site of infection (7). As AMB penetrates the central nervous system (CNS) poorly, intraventricular AMB has been used successfully in severe cases of cryptococcal meningitis under the premise that local administration will increase its concentration within the CNS (8). However, this approach commonly leads to CNS complications and tolerance (3).

The patient with $C$. neoformans in the current case study had poor tolerance for intravenous administration of liposomal AmB, and symptoms were not relieved following 5 months treatment with $150 \mathrm{mg}$ /day liposomal AmB. In order to rapidly control the intracranial infection, continuous intrathecal administration of liposomal AmB was conducted over 1 month.

\section{Case report}

The present report was approved by the Ethics Committee of Ethical Research for Zhujiang Hospital of Southern Medical University (Guangzhou, China). The patient provided consent for the case and related images to be used in this report. 
A 28-year-old male patient, was admitted to the Department of Neurology, Zhujiang Hospital of Southern Medical University on 10 May 2014, having experienced a headache and fever for $>20$ days. From 16 April, the patient had begun experiencing headaches without obvious inducement, mainly presenting with paroxysmal aggravated headache. The patient had no symptoms of dizziness, nausea, vomiting, unsteady walking, limbs lacking in strength, numbness of limbs, convulsion of limbs, unconsciousness, hearing loss, blurred vision or double vision. Following 3 days of headache, the patient developed a low-grade fever, with a maximum temperature of $37.5^{\circ} \mathrm{C}$. The patient sought treatment at a local hospital, where the disease was regarded as an upper respiratory infection. However, the patient did not get better after receiving treatment suitable for this diagnosis (dextromethorphan hydrobromide tablets; 30 mg twice daily; Guangzhou Baiyun Shan Guanghua Pharmaceutical Co., Ltd., Guangzhou, China; and Xiaochaihu granules, Guangzhou Baiyun Shan Guanghua Pharmaceutical Co., Ltd.; $10 \mathrm{~g}$ twice daily) and the patient's condition continued to decline. The patient attended PANYU Central Hospital (Guangzhou, China) in May 2014 for treatment. A routine blood examination indicated that the white blood cell was $13 \times 10^{9}$ cells/1 (reference value $4-10 \times 10^{9}$ cells/1). The electroencephalogram presented as slightly abnormal, with decreased alpha wave and increased theta wave activity. Symptoms did not improve following treatment with piperacillin (4 g intravenously twice daily) during admission, and the patient experienced two occurrences of foaming at the mouth, upturning binoculus and convulsion of limbs on 5 and 6 May 2014. Following administration with tranquilizing drugs including diazepam (Harbin Aurora Optoelectronic Technology Co., Ltd., Harbin, China; 10 mg intramuscular injection, twice daily) and phenobarbital sodium (Guangdong Bangmin Pharmaceutical Co., Ltd., Jianmen, China; 100 mg intramuscular injection, twice daily), the epilepsy torsion symptoms were alleviated; however, the headache continuously intensified. The patient also experienced low-grade fever and gradual clouding of consciousness. The patient attended an appointment on 10 May 2014 at the Neurology Department of Zhujiang Hospital of Southern Medical University and was admitted with 'intracranial infection'. Lumbar puncture was administered to the patient and the tested pressure of cerebrospinal fluid was $350 \mathrm{mmH}_{2} \mathrm{O}$. The cerebrospinal fluid tested positive for the antigen of Cryptococcus (Fig. 1). C. neoformans was observed with ink staining.

The patient was treated with liposomal AmB, with an initial dose of $0.1 \mathrm{mg} / \mathrm{kg}$ increasing up to $2.5 \mathrm{mg} / \mathrm{kg}$ daily by intravenous injection and combined use of oral flucytosine tablets (China Pharmaceutical University Pharmaceutical Co., Ltd., Nanjing, China; 1.5 g/day) and fluconazole (0.4 g/day; Pfizer, Inc., New York, NY, USA) through intravenous drip. Dehydration was also induced to reduce intracranial pressure. Through these treatments, the patient's temperature returned to normal, and headache did not get worse, but the patient still had blurred vision. C. neoformans was still observed following repeated lumbar puncture, but when the dosage of liposomal AmB reached $150 \mathrm{mg} /$ day, the patient experienced considerable discomfort, including xanthochromia, pruritus, poor appetite and drowsiness, thus the dosage could not be increased.
Following administration of this treatment for 5 months, C. neoformans could still be detected in the cerebrospinal fluid. Therefore, it was decided that continuous intrathecal administration of liposomal AmB should be conducted. On 13 November 2014, a Medtronic lumbar puncture external drainage suite (product model 27303; Medtronic, Minneapolis, MN, USA) was used. The space between 3/4 lumbar vertebrae was selected, $20 \mathrm{~cm}$ of the head end of the drainage tube was inserted; then 30-50 ml cerebrospinal fluid was slowly discharged prior to each administration of the drug. For 1 month, liposomal AmB was administered for 20 h each day; an additional $4 \mathrm{~h}$ was required to slowly discharge cerebrospinal fluid and change the dressing. The dosage of liposomal AmB was increased by $0.5 \mathrm{mg}$ each day. When the dosage reached $2 \mathrm{mg} /$ day, the patient started to exhibit damage to the oculomotor nerve on both sides and damage to the facial nerve on the left side. The dosage was subsequently decreased to $1 \mathrm{mg} /$ day, after which the cranial nerve damage gradually recovered, headache was reduced fully and temperature remained normal. The patient received a total of $28 \mathrm{mg}$ intrathecal liposomal Amb over the course of the month.

Following the removal of the drainage tube on the lumbar cistern, six consecutive ink stains of cerebrospinal fluid tested negative for Cryptococcus. As indicated in Fig. 1, in the reexamination on 23 November 2014, a relatively large number of lymphocytes were observed in the hematoxylin and eosin stain of cerebrospinal fluid, and no C. neoformans could be detected (observed using light microscopy). The patient was discharged on 6 February 2015. Follow-up visits were conducted for 6 months and no reappearance of $C$. neoformans was observed.

As presented in Table I, the patient's cerebrospinal fluid was tested 30 times during the hospitalization period. The cerebrospinal fluid in the 20th-24th examinations were samples kept from the drainage tube on the lumbar cistern. Magnetic resonance imaging scan and enhanced images of the patient's brain on day 3 after admission (in May 2014) and the day before leaving hospital (in February 2015) are presented in Fig. 2.

Cerebrospinal fluid pressure was $325.79 \pm 34.85 \mathrm{~mm} \mathrm{H}_{2} \mathrm{O}$, white blood cell count was $26.63 \pm 29.16 \times 10^{6}$ cells $/ 1$ and total cerebrospinal fluid protein was $612.53 \pm 209.74 \mathrm{mg} / \mathrm{l}$. After the continuous intrathecal administration of liposomal amphotericin $\mathrm{B}$, the cerebrospinal fluid pressure was $252.50 \pm 45.36 \mathrm{~mm}$ $\mathrm{H}_{2} \mathrm{O}$, the white blood cell count was $20 \pm 8.94 \times 10^{6}$ cells $/ 1$ and total cerebrospinal fluid protein was $450 \pm 65.49 \mathrm{mg} / 1$. These results suggest that administration of amphotericin $B$ liposomes caused cerebrospinal fluid levels and increased intracranial hypertension to gradually return to normal, and the white blood cell levels and the total cerebrospinal fluid protein were decreased.

\section{Discussion}

The Clinical Practice Guidelines for Cryptococcosis (4) (hereinafter referred to as 'Guidelines'), which were updated by the Infectious Disease Society of America in 2010, suggest using AmB deoxycholic acid (AmBd; 0.7-1.0 mg/kg/day, via intravenous drip) and flucytosine (100 mg/kg/day, orally) for inductive treatment for at least 4 weeks, followed by treatment with fluconazole (400 mg/day) for 8 weeks for patients with 
Table I. Cerebrospinal fluid pressure and results of laboratory tests.

\begin{tabular}{|c|c|c|c|c|c|c|c|}
\hline Time & $\begin{array}{c}\text { Date } \\
(\mathrm{dd} / \mathrm{mm} / \text { yyyy })\end{array}$ & $\begin{array}{c}\text { Cerebrospinal fluid } \\
\text { pressure } \\
\left(\mathrm{mm} \mathrm{H}_{2} \mathrm{O}\right)\end{array}$ & $\begin{array}{l}\text { White blood cell } \\
\text { count } \\
\left(10^{6} \text { cells } / \mathrm{lM} / \mathrm{l}\right)\end{array}$ & $\begin{array}{l}\text { Chloride ion } \\
(\mathrm{mmol} / \mathrm{l})\end{array}$ & $\begin{array}{l}\text { Glucose } \\
(\mathrm{mmol} / \mathrm{l})\end{array}$ & $\begin{array}{l}\text { Lactic acid } \\
(\mathrm{mmol} / \mathrm{l})\end{array}$ & $\begin{array}{l}\text { Total cerebrospinal } \\
\text { fluid protein } \\
(\mathrm{mg} / \mathrm{l})\end{array}$ \\
\hline 1 & $10 / 05 / 2014$ & 350 & 50 & 118.2 & 2.5 & 2.5 & 459 \\
\hline 2 & $27 / 05 / 2014$ & 300 & 50 & 117.7 & 2.0 & 3.3 & 666 \\
\hline 3 & 09/06/2014 & 400 & 50 & 115.7 & 1.4 & 3.6 & 926 \\
\hline 4 & $20 / 06 / 2014$ & 300 & 70 & 114.4 & 1.6 & 3.6 & 914 \\
\hline 5 & 03/07/2014 & 320 & 110 & 118.6 & 3.4 & 3.1 & 541 \\
\hline 6 & $22 / 07 / 2014$ & 350 & 25 & 121.6 & 3.8 & 2.5 & 562 \\
\hline 7 & $30 / 07 / 2014$ & 350 & 20 & 117.7 & 3.9 & 2.2 & 425 \\
\hline 8 & 07/08/2014 & 350 & 20 & 116.2 & 2.6 & 1.5 & 398 \\
\hline 9 & $16 / 08 / 2014$ & 330 & 4 & 118.5 & 3.8 & 1.5 & 465 \\
\hline 10 & $22 / 08 / 2014$ & 250 & 9 & 122 & 3.9 & 2.3 & 513 \\
\hline 11 & $29 / 08 / 2014$ & 300 & 0 & 124.2 & 3.8 & 2.1 & 519 \\
\hline 12 & 04/09/2014 & 320 & 12 & 120.5 & 3.8 & 2.2 & 558 \\
\hline 13 & $04 / 09 / 2014$ & 320 & 2 & 121 & 3.4 & 2.7 & 633 \\
\hline 14 & $18 / 09 / 2014$ & 320 & 1 & 122.9 & 3.6 & 2.5 & 565 \\
\hline 15 & $25 / 09 / 2014$ & 320 & 3 & 124.7 & 3.4 & 2.8 & 673 \\
\hline 16 & 08/10/2014 & 350 & 30 & 123.1 & 3.7 & 2.9 & 475 \\
\hline 17 & $15 / 10 / 2014$ & 260 & 10 & 123.6 & 3.8 & 2.3 & 522 \\
\hline 18 & $23 / 10 / 2014$ & 350 & 0 & 121.7 & 3.6 & 2.0 & 572 \\
\hline 19 & $11 / 11 / 2014$ & 350 & 40 & 124.2 & 3.0 & 2.7 & 1252 \\
\hline 20 & $19 / 11 / 2014$ & - & 50 & 111.7 & 5.6 & 7.4 & 382 \\
\hline 21 & $21 / 11 / 2014$ & - & 20 & 130.8 & 4.8 & 4.1 & 209 \\
\hline 22 & $23 / 11 / 2014$ & - & 50 & 126.3 & 4.1 & 3.4 & 754 \\
\hline 23 & $10 / 12 / 2014$ & - & 30 & 120.1 & 3.0 & 2.6 & 293 \\
\hline 24 & 08/12/2014 & - & 0 & 122.2 & 2.6 & 2.5 & 422 \\
\hline 25 & $18 / 12 / 2014$ & 320 & 30 & 122.6 & 2.7 & 2.7 & 534 \\
\hline 26 & $24 / 12 / 2014$ & 300 & 20 & 124.3 & 2.7 & 3.0 & 516 \\
\hline 27 & $30 / 12 / 2014$ & 220 & 10 & 122.8 & 2.8 & 3.2 & 463 \\
\hline 28 & 06/01/2015 & 230 & 20 & 125.0 & 2.5 & 3.2 & 388 \\
\hline 29 & $13 / 01 / 2015$ & 215 & 30 & 122.5 & 2.9 & 2.6 & 421 \\
\hline 30 & $19 / 01 / 2015$ & 230 & 10 & 120.9 & 3.3 & 1.6 & 378 \\
\hline
\end{tabular}

The cerebrospinal fluid in examinations 20-24 was a sample kept from the drainage tube on the lumbar cistern, for which the pressure could not be tested.

normal immunological functions. If patients cannot tolerate AmBd, treatment with liposomal AmB (3-4 mg/ $\mathrm{kg} /$ day, intravenous drip) or AmB lipid complex (5 mg/kg/day, intravenous drip) should be conducted (4). In the current case study, the patient could not tolerate an expected dosage of liposomal $\mathrm{AmB}$, and the dose administered did not reach the target dose of 3-4 mg/kg/day. This suggests that liposomal AmB did not reached the minimal inhibitory concentration over the 5-month treatment period. Although three types of drug were administered and the clinical symptoms of the patient stabilized, Cryptococcus was still present and the disease had a protracted course.

Damage to the cranial nerve occurred when the intrathecal dosage of liposomal AmB was increased to $2 \mathrm{mg}$ /day. When the dosage was decreased to $1 \mathrm{mg} / \mathrm{day}$, the damage to the cranial nerve was reduced and patient experienced an effective reduction of symptoms. It has been proposed that during the circulation of cerebrospinal fluid of the patient, the drug is distributed in the cerebrospinal fluid and is absorbed into the blood along with arachnoid granulations. In an infected state, the rate and quantity of absorption of arachnoid granulations may have significant individualized differences and the simple application of the drug metabolism rule during cerebrospinal fluid circulation under physiological status is not appropriate $(9,10)$. Hence, it is proposed that a safe dosage of liposomal AmB should begin at $0.5 \mathrm{mg} / \mathrm{day}$, increasing by $0.5 \mathrm{mg} /$ day every $1-2$ days, with close monitoring of any damage to the cranial nerve and etiological examination of cerebrospinal fluid, based on which an individualized concentration of liposomal AmB can be determined.

The Guidelines do not recommend intrathecal injection of liposomal $\mathrm{AmB}$, as the interval time of intrathecal injection 


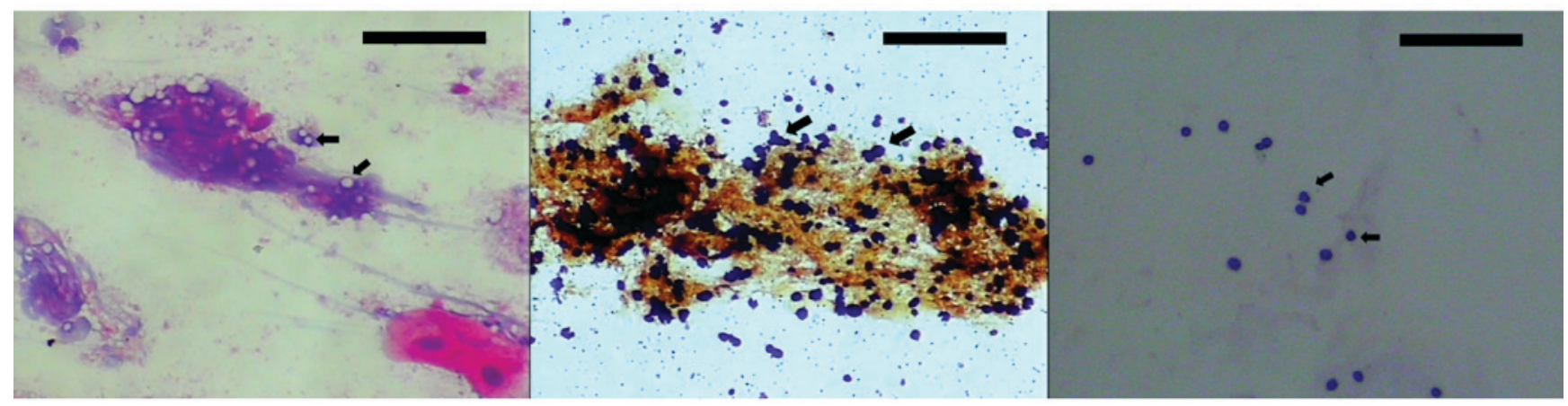

Figure 1. (A) Hematoxylin-eosin stain of cerebrospinal fluid, indicating a large number of round hyaline bodies, which were Cryptococcus neoformans. Magnification, x400. (B) Methenamine silver stain of cerebrospinal fluid. Magnification, x400. (C) Hematoxylin-eosin staining of cerebrospinal fluid, indicating a relatively large number of lymphocytes. Scale bars $=200 \mathrm{~nm}$.
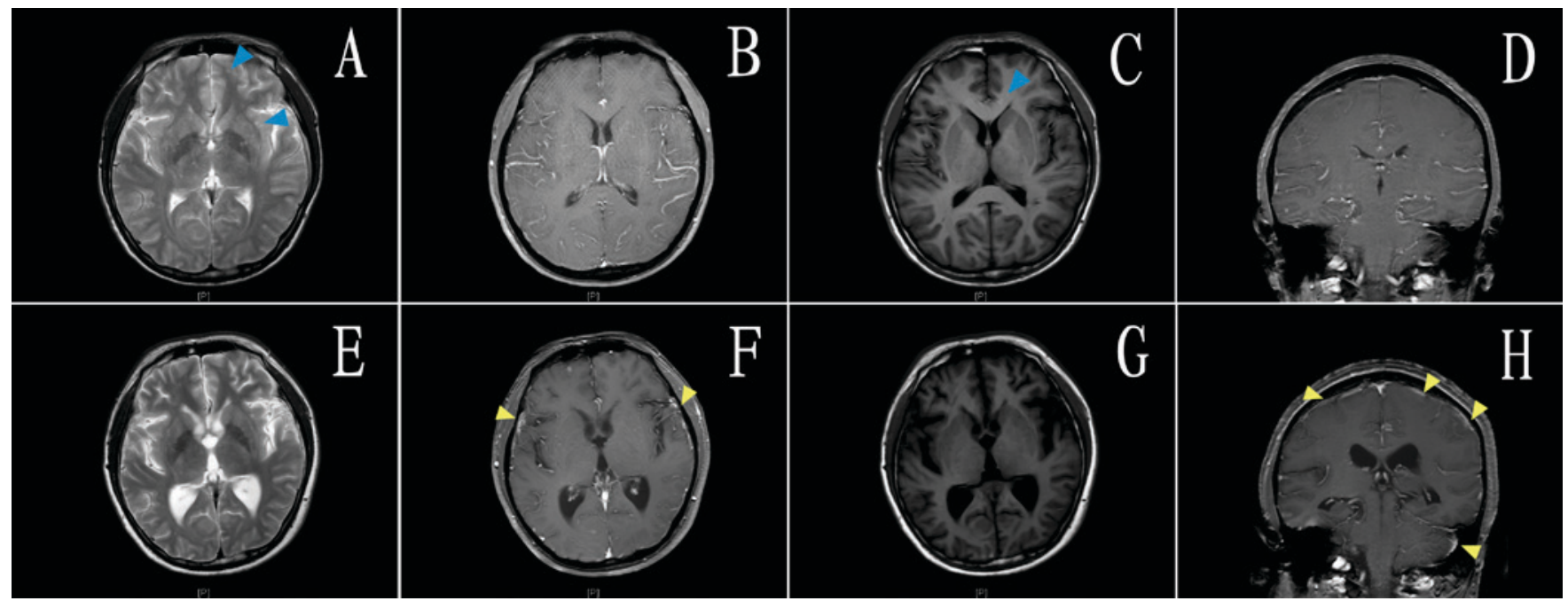

Figure 2. (A and B) MRI scans and (C and D) enhanced images of the patient's brain prior to treatment, displaying the bilateral parietal cortex, the left insular lobe, the right lenticular nucleus and right cornu anterius ventriculi lateralis as slightly spot or patchy long T1 and long T2 hyperintense (blue arrows). (E and F) MRI scans and (G and H) enhanced images of the patient's brain following treatment on 04/01/2015. The bilateral frontal gyrus became nodular or patchy; the meninges of front temporal pole (especially at the right side) were displayed as a narrow band and became thickened and enhanced (yellow arrow); bilateral temporal and cerebral ventricle bodies were symmetrically expanded. MRI, magnetic resonance imaging.

is too long (4). It is also impossible to form a stable drug concentration in the cerebrospinal fluid - this is unsafe and does not help with controlling the infection. The current authors propose that continuous intrathecal administration may compensate for the weaknesses of conventional intrathecal administration in the following ways: i) The duration and interval of administration may be more effectively controlled; ii) extreme peaks in drug concentration are avoided, reducing the risk of a toxic reaction of the nervous system occurring; and iii) the stable drug concentration can control the infection more effectively.

The course of treatment for continuous intrathecal administration was 1 month. Cerebrospinal fluid can easily effuse from the sinus tract during puncture, which may lead to catheter-related infections $(11,12)$. However, this risk can be reduced by careful disinfection by iodine tincture and ethyl alcohol every day. Prior to each continuous intrathecal administration, 30-50 $\mathrm{ml}$ cerebrospinal fluid should be slowly discharged to relieve the increase in cerebrospinal fluid pressure. If any necrosis at the pipe orifice is observed, the catheter should be removed immediately and a new catheter placed at the space between other lumbar vertebrae. If infection symptoms appear, broad-spectrum antibiotics with high efficiency should be used immediately to control the infection (13).

In conclusion, conventional intravenous administration may be suitable for most $C$. neoformans encephalitis cases, although continuous intrathecal administration is not recommended for all patients. However, if an increasing dosage of liposomal AmB is not tolerated by the patient, continuous intrathecal administration is an effective way to treat patients with refractory $C$. neoformans encephalitis.

\section{References}

1. Furukawa K, Sasaki H, Pollard RB and Suzuki F: Lanoconazole, a new imidazole antimycotic compound, protects MAIDS mice against encephalitis caused by Cryptococcus neoformans. J Antimicrob Chemother 46: 443-450, 2000. 
2. Saag MS, Powderly WG, Cloud GA, Robinson P, Grieco MH, Sharkey PK, Thompson SE, Sugar AM, Tuazon CU, Fisher JF, et al: Comparison of amphotericin B with fluconazole in the treatment of acute AIDS-associated cryptococcal meningitis. N Engl J Med 326: 83-89, 1992.

3. Mitchell TG and Perfect JR: Cryptocoecosis in the era of AIDS-100 years after the discovery of Cryptococcus neoformans. Clin Microbiol Rev 8: 515-548, 1995.

4. Desalermos A, Kourkoumpetis TK and Mylonakis E: Update on the epidemiology and management of cryptococcal meningitis. Expert Opini Pharmacother 13: 783-789, 2012.

5. Levitz SM: The ecology of Cryptococcus neoformans and the epidemiology of cryptococcosis. Rev Infect Dis 13: 1163-1169, 1991.

6. Coker RJ, Viviani M, Gazzard BG, Du Pont B, Pohle HD, Murphy SM, Atouguia J, Champalimaud JL and Harris JR: Treatment of cryptococcosis with liposomal amphotericin B (AmBisome) in 23 patients with AIDS. Aids 7: 829-835, 1993.

7. Mwaba P, Mwansa J, Chintu C, Pobee J, Scarborough M, Portsmouth S and Zumla A: Clinical presentation, natural history, and cumulative death rates of 230 adults with primary cryptococcal meningitis in Zambian AIDS patients treated under local condition. Postgrad Med J 77: 769-773, 2001.
8. Pappas PG, Kontoyiannis DP, Perfect JR and Chiller TM Real-time treatment guidelines: Considerations during the Exserohilum rostratum outbreak in the United States. Antimicrob Agents Chemother 57: 1573-1576, 2013.

9. Greitz D and Hannerz J: A proposed model of cerebrospinal fluid circulation: Observations with radionuclide cisternography. AJNR Am J Neuroradiol 17: 431-438, 1996.

10. Brinker T, Stopa E, Morrison J and Klinge P: A new look at cerebrospinal fluid circulation. Fluids Barriers CNS 11: 10, 2014.

11. Lemaire X, Morena M, Leray-Moragués H, Henriet-Viprey D, Chenine L, Defez-Fougeron C and Canaud B: Analysis of risk factors for catheter-related bacteremia in 2000 permanent dual catheters for hemodialysis. Blood Purif 28: 21-28, 2009.

12. Jean G, Charra B, Chazot C, Vanel T, Terrat JC, Hurot JM and Laurent G: Risk factor analysis for long-term tunneled dialysis catheter-related bacteremias. Nephron 91: 399-405, 2002.

13. Weber DJ and Rutala WA: Central line-associated bloodstream infections: Prevention and management. Infect Dis Clin North Am 25: 77-102, 2011. 